\title{
Theoretical mass loss rates of cool main-sequence stars
}

\author{
V. Holzwarth ${ }^{1,2}$ and M. Jardine ${ }^{2}$ \\ 1 Max-Planck-Institut für Sonnensystemforschung, Max-Planck-Strasse 2, 37191 Katlenburg-Lindau, Germany \\ e-mail: holzwarth@mps .mpg. de \\ 2 School of Physics and Astronomy, University of St Andrews, North Haugh, St Andrews, Fife KY16 9SS, Scotland \\ e-mail: mmj@st-andrews.ac.uk
}

Received 8 November 2006 / Accepted 26 October 2006

\section{ABSTRACT}

\begin{abstract}
Context. The stellar mass loss rate is important for the rotational evolution of a star and for its interaction with the circumstellar environment. The analysis of astrospheric absorption features enables an empirical determination of mass loss rates of cool stars other than the Sun.

Aims. We develop a model for the wind properties of cool main-sequence stars, which comprises their wind ram pressures, mass fluxes, and terminal wind velocities.

Methods. The wind properties are determined through a polytropic magnetised wind model, assuming power laws for the dependence of the thermal and magnetic wind parameters on the stellar rotation rate. We use the empirical data to constrain theoretical wind scenarios, which are characterised by different rates of increase of the wind temperature, wind density, and magnetic field strength. Results. Scenarios based on moderate rates of increase yield wind ram pressures in agreement with most empirical constraints, but cannot account for some moderately rotating targets, whose high apparent mass loss rates are inconsistent with observed coronal $\mathrm{X}$-ray and magnetic properties. For fast magnetic rotators, the magneto-centrifugal driving of the outflow can produce terminal wind velocities far in excess of the surface escape velocity. Disregarding this aspect in the analyses of wind ram pressures leads to overestimations of stellar mass loss rates. The predicted mass loss rates of cool main-sequence stars do not exceed about ten times the solar value.

Conclusions. Our results are in contrast with previous investigations, which found a strong increase of the stellar mass loss rates with the coronal X-ray flux. Owing to the weaker dependence, we expect the impact of stellar winds on planetary atmospheres to be less severe and the detectability of magnetospheric radio emission to be lower then previously suggested. Considering the rotational evolution of a $1 M_{\odot}$ star, the mass loss rates and the wind ram pressures are highest during the pre-main sequence phase.
\end{abstract}

Key words. stars: winds, outflows - stars: mass-loss - stars: magnetic fields - stars: late-type - stars: planetary systems

\section{Introduction}

Solar-like stars with hot coronae are expected to lose mass in the form of stellar winds (Parker 1960). In contrast to the P Cygni line profiles of the massive winds of hot stars and young T Tauri stars (e.g. Dupree et al. 2005), the tenuous and highly ionised outflows of cool stars yield no detectable radiative signatures and cannot be diagnosed directly. Wood et al. (2002) devised an indirect method to deduce mass loss rates of cool stars from specific properties of the astrospheres blown into the ambient interstellar medium by their stellar wind (see also Wood \& Linsky 1998; and Wood 2004). Hot neutral hydrogen in the heliosphere, mainly in the form of a $\mathrm{H}$ I wall between the heliopause and the bow shock, leads to the formation of a red-shifted absorption feature in stellar Ly $\alpha$ emission lines, whereas a corresponding structure in the astrosphere around a star causes a specific blue-shifted feature. The size and absorption capabilities of the astrosphere depend on the strength of the stellar wind as well as on the properties (i.e. density, relative velocity) of the ambient interstellar medium (ISM).

By fitting synthetic absorption features, obtained from hydrodynamical simulations of the wind-ISM interaction (e.g. Zank et al. 1996; Müller et al. 2001), to observed line profiles, Wood et al. (2002, 2005a) estimate the wind ram pressures and mass loss rates of cool stars in the solar neighbourhood. Owing to the requirement of accurate ISM properties, which are only available for the solar vicinity, the sample of observed stars is currently small and rather heterogeneous, comprising single and binary stars of different spectral types and luminosity classes. For $\mathrm{G}$ and $\mathrm{K}$ main-sequence stars with $\mathrm{X}$-ray fluxes $F_{\mathrm{X}} \lesssim 8 \times 10^{5} \mathrm{erg} \mathrm{s}^{-1} \mathrm{~cm}^{-2}$, Wood et al. find a correlation between the coronal activity and the deduced mass loss rate per stellar surface area, $\dot{M} / R^{2} \propto F_{\mathrm{X}}^{1.34 \pm 0.18}$. Since the more active stars of the sub-sample have mass loss rates lower than predicted by this power-law relation, Wood et al. speculate that this may be due to a change of the stellar magnetic field topology.

The $\dot{M}-F_{\mathrm{X}}$-relationships of Wood et al. (2002, 2005a) have been applied to the rotational evolution of cool stars and to their wind interaction with extra-solar planets. Wood et al. (2002) extrapolate the mass loss history of solar-like stars backward in time and suggest that the mass loss rate of the young Sun may have been up to three orders of magnitude higher than today. Considering the "faint young Sun" paradox, that is the apparently unchanged planetary temperatures despite an about $25 \%$ less luminous Sun about 3.8 Gyr ago (e.g. Gaidos et al. 2000; Shaviv 2003, and references therein), they find that the cumulated mass loss of about $0.03 M_{\odot}$ can not account for the $\sim 10 \%$ difference in solar mass, which has been suggested to solve this problem in terms of a more luminous, higher-mass young Sun. Grießmeier et al. (2004) investigate the particle and thermal losses from the upper atmospheres of "Hot Jupiters" and 
find that high stellar mass loss rates have a significant influence on the evolution of the planetary mass and radius. The strength of stellar winds also affects the detectability of extra-solar giant planets in radio wavelengths, since the emission scales with the kinetic and magnetic energy flux on the planetary magnetosphere (e.g. Stevens 2005; Grießmeier et al. 2005). Owing to the high predicted stellar wind ram pressures, the magnetospheric radio emission levels of close-by planets are expected to be sufficiently high to become detectable with future instruments.

Wood et al. (2002, 2005a) use scaled versions of the solar wind to match observed and simulated line profiles. With their principal quantity being the wind ram pressure, they presume the terminal wind velocity of all stars to be solar-like, so that the wind density is the only free fitting parameter. The presumption of thermally driven winds with a unique terminal wind velocity neglects, however, the influence of the magnetic field on the acceleration and structuring of the outflow. Coronal activity signatures and the magnetic braking of cool main-sequence stars are indicative for surface magnetic flux, which is generated by dynamo processes within the outer convection zone. The magnetic field gives rise to the formation of hot X-ray emitting coronal loops as well as to the acceleration of plasma escaping along open field lines, and links ab initio the two quantities in the power law suggested by Wood et al. (2005a).

We consider the impact of magnetic fields in more detail by determining wind ram pressures and mass loss rates in the framework of a magnetised wind model. Using the mass loss rates inferred by Wood et al. (2002, 2005a) as empirical constraints for possible wind scenarios, our working hypothesis is that the magnetic and thermal wind properties of cool stars primarily depend on the stellar rotation rate. In Sect. 2, we describe the magnetised wind model, the principal quantities of the investigation, and the observational constraints of the model parameters. Section 3 comprises the analyses of characteristic wind properties resulting from different wind scenarios, and the comparisons of theoretical and empirical wind ram pressures and mass loss rates. In Sect. 4, we discuss our results and their consequences for the evolution of stellar rotation and mass loss and for the detectability of extra-solar planetary magnetospheres. Our conclusions are summarised in Sect. 5.

\section{Model considerations}

\subsection{Magnetised wind model}

We consider main-sequence stars with masses $0.2 M_{\odot} \leq M \leq$ $1.2 M_{\odot}$, radii $R \propto M^{0.8}$, and rotation rates $0.6 \Omega_{\odot} \leq \Omega \leq 11.3 \Omega_{\odot}$; with the solar rotation rate $\Omega_{\odot}=2.8 \times 10^{-6} \mathrm{~s}^{-1}$ the stellar rotation periods are between $2.3 \mathrm{~d}$ and $43 \mathrm{~d}$. The winds are determined in the framework of the magnetised wind model of Weber \& Davis (1967). The properties of stationary, axisymmetric, and polytropic outflows are specified through the wind temperature, $T_{0}$, the wind density, $\rho_{0}$, and the radial magnetic field strength, $B_{0}$, at a reference level, $r_{0}=1.1 \mathrm{R}$, close to the stellar surface. The model solutions provide the flow velocity, $v_{\mathrm{r}, \mathrm{A}}$, and the density, $\rho_{\mathrm{A}}$, of the outflowing plasma at the Alfvénic radius, $r_{\mathrm{A}}$, where the flow velocity equals the local Alfvén velocity (Holzwarth 2005). The location of and the conditions at the Alfvénic point specify the wind structure along a magnetic field line as well as the mass loss rate (see, e.g., Lamers \& Cassinelli 1999; Mestel 1999).

We assume that the entire stellar surface contributes to the wind. Closed loop-like magnetic fields structures reduce the effective surface area from which outflows can emanate and also

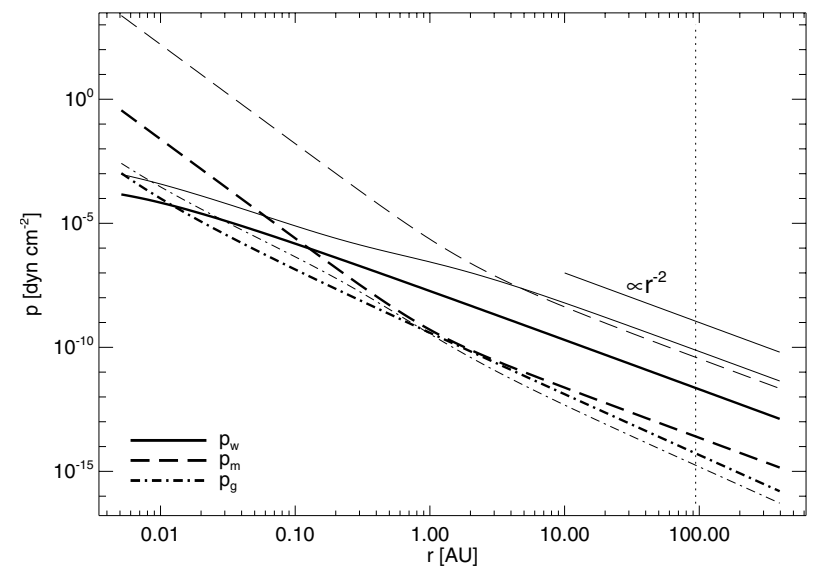

Fig. 1. Radial profiles of the ram pressure, $p_{\mathrm{w}}$, the magnetic pressure, $p_{\mathrm{m}}$, and the thermal gas pressure, $p_{\mathrm{g}}$, of magnetised winds in the equatorial plane. For solar wind parameters (thick lines), $T_{0}=$ $2.93 \times 10^{6} \mathrm{~K}, n_{0}=2.76 \times 10^{6} \mathrm{~cm}^{-3}$, and $B_{0}=3 \mathrm{G}$, the magnetic and thermal pressures at the heliospheric termination shock (dotted line) are over two orders of magnitude smaller than the wind ram pressure. Thin lines show the pressure profiles of a fast magnetic rotator with a rotation period of six days and wind parameters $T_{0}=3.4 \times 10^{6} \mathrm{~K}, n_{0}=$ $6.67 \times 10^{6} \mathrm{~cm}^{-3}, B_{0}=243 \mathrm{G}$. At large distances from the star the wind ram pressure decreases $\propto r^{-2}$. Note that it is not the magnetic pressure which gives rise to the magneto-centrifugal acceleration of the wind, but the stellar rotation in conjunction with the plasma outflow along bent magnetic field lines.

influence the flow structure in adjacent wind zones (e.g. Mestel 1968; Priest \& Pneuman 1974; Kawaler 1988). The different magnetic field topologies encountered during a solar activity cycles entail, for example, mass loss variations within a factor of 2 (Wang 1998). Closed-field regions are however limited to a few stellar/solar radii above the surface, well below the Alfvénic surface (e.g. Jardine et al. 2002a,b). We expect that small-scale spatial and short-term temporal inhomogeneities are averaged out with increasing distance from the star and thus irrelevant for the sustainment of astrospheres.

\section{2. "Characteristic" wind ram pressure}

The mass loss rate along a slender open magnetic flux tube is

$\mathrm{d} \dot{M}=F_{\mathrm{m}} \mathrm{d} \sigma$,

where $\mathrm{d} \sigma=\sin \theta \mathrm{d} \theta \mathrm{d} \phi$ is the solid angle occupied by the flux tube. The mass flux per solid angle, $F_{\mathrm{m}}=\rho v_{r} r^{2}=\rho_{\mathrm{A}} v_{r, \mathrm{~A}} r_{\mathrm{A}}^{2}$, is constant along an individual tube. The rate of the momentum transport associated with the mass flux,

$\mathrm{d} \dot{M} v_{r}=\rho v_{r}^{2} r^{2} \mathrm{~d} \sigma=p_{\mathrm{w}} \mathrm{d} S$,

is equivalent to the force which results from the wind ram pressure, $p_{\mathrm{w}}=\rho v_{r}^{2}$, exerted on the local tube cross section, $\mathrm{d} S=r^{2} \mathrm{~d} \sigma$. At large distances from the star the outflow velocity converges to the constant terminal velocity, $v_{\infty}$. In contrast to the wind ram pressure, which decreases $\propto r^{-2}$ (Fig. 1), the ram force per solid angle,

$\frac{\mathrm{d} \mathcal{F}_{\mathrm{w}}}{\mathrm{d} \sigma}=p_{\mathrm{w}} r^{2}=F_{\mathrm{m}} v_{\infty}=$ const.

is independent of the radius. This quantity comprises both the wind density and (terminal) wind velocity, and enables a characterisation of the mass loss rate of individual stars. In the case 
of spherically symmetric outflows, for which $\mathrm{d} \sigma=4 \pi$, the ram force is $\mathcal{F}_{\mathrm{w}}=4 \pi F_{\mathrm{m}} v_{\infty}=\dot{M} v_{\infty}$. To compare wind properties of stars with different stellar radii, we use the ram force per stellar surface area,

$\mathcal{P}=\frac{\mathcal{F}_{\mathrm{w}}}{4 \pi R^{2}}=\frac{\dot{M} v_{\infty}}{4 \pi R^{2}}=\frac{F_{\mathrm{m}} v_{\infty}}{R^{2}}$,

as the principal quantity of our investigation and shall refer to it as the characteristic wind ram pressure (CWRP).

\subsection{Empirical wind ram pressures}

In their analysis of astrospherical absorption features, Wood et al. (2002, 2005a) adopt for all stars in their sample solar-like winds with a unique terminal velocity of $400 \mathrm{~km} \mathrm{~s}^{-1}$. This assumption implies that the relative CWRP (in solar units),

$\frac{\mathcal{P}}{\mathcal{P}_{\odot}}=\frac{\dot{M}}{\dot{M}_{\odot}} \frac{v_{\infty}}{v_{\infty, \odot}}\left(\frac{R}{R_{\odot}}\right)^{-2}$,

is equivalent to the stellar mass loss rate per surface area:

$\left(\frac{\mathcal{P}}{\mathcal{P}_{\odot}}\right)_{\mathrm{W}}=\left(\frac{\dot{M}}{\dot{M}_{\odot}}\right)_{\mathrm{W}}\left(\frac{R}{R_{\odot}}\right)^{-2} \quad$ for $v_{\infty}=v_{\infty, \odot}$.

In conjunction with individual stellar radii, the empirical mass loss rates, $\dot{M}_{\mathrm{W}}$, given by Wood et al. $(2002,2005$ a), provide the observational constraints for our theoretical CWRPs.

\subsection{Rotation-dependent wind parameters}

The activity levels of cool stars increase with the stellar rotation rate (e.g. Noyes et al. 1984), comprising enhanced thermal and magnetic field values inside the coronae. We take this aspect into account by assuming that the wind parameters follow power-law relations, which are based on the solar reference case.

The rates of increase of the thermal wind parameters ${ }^{1}$

$T_{0}=T_{0, \odot}\left(\frac{\Omega}{\Omega_{\odot}}\right)^{n_{T}} \quad$ and $\quad n_{0}=n_{0, \odot}\left(\frac{\Omega}{\Omega_{\odot}}\right)^{n_{n}}$

at the reference level are specified through the power-law indices $n_{T}$ and $n_{n}$, respectively. Polarimetric observations indicate that the magnetic flux depends on the stellar rotation rate (e.g. Saar 1991). Taking different stellar radii (here, $R \propto M^{0.8}$ ) into account, the magnetic field strengths are taken to follow the powerlaw relation

$B_{0}=B_{0, \odot}\left(\frac{M}{M_{\odot}}\right)^{-1.6}\left(\frac{\Omega}{\Omega_{\odot}}\right)^{n_{\Phi}}$,

which implies that for a given stellar rotation rate lower-mass stars have higher average field strengths.

The assumption of polytropic magnetised winds whose wind parameters follow simple power-laws may be simplistic, but in view of the currently limited observational constraints a more sophisticated model appears inappropriate.

${ }^{1}$ For consistency reasons, we use the particle density, $n_{0}=\rho_{0} N_{\mathrm{A}} / \mu$, to specify the wind condition at the reference level, with the mean molecular weight $\mu$ and the Avogadro number $N_{\mathrm{A}}$.

\subsubsection{Observational constraints}

Since the thermal wind properties of cool stars cannot be observed directly, we follow the hypothetical assumption that closed coronal magnetic field regions may serve as proxies to constrain the increase of the temperature and density with the stellar rotation rate. Analysing the dependence of stellar X-ray luminosities, Ivanova \& Taam (2003) infer a density power-law index $n_{n} \approx 0.6$, which implies for stars rotating ten times faster than the Sun (i.e. close to the X-ray saturation limit) about four times higher coronal densities. Differential emission measures of rapidly rotating stars indicate a large fraction of plasma with temperatures of $\sim 10^{7} \mathrm{~K}$, in addition to solar-like coronal plasma components with temperatures $\gtrsim 10^{6} \mathrm{~K}$ (e.g. Favata \& Micela 2003, and references therein). A coronal temperature of $10 \mathrm{MK}$ for a rapidly rotating star like $\mathrm{AB} \operatorname{Dor}\left(\Omega \simeq 50 \Omega_{\odot}\right)$ would imply a temperature power-law index of $n_{T} \simeq 0.5$. Following the solar paradigm, it is more likely that the temperature of stationary stellar winds are characterised by the low-temperature plasma component, implying values $0 \lesssim n_{T}<0.5$.

The increase of the magnetic flux with the stellar rotation rate is constrained through direct magnetic flux measurements (e.g. Saar 1991, 2001, and references therein) and, indirectly, through empirical activity-rotation-age relations. Due to the braking effect of magnetised winds, solar-like single stars spin down during the course of their main-sequence evolution. In conjunction with the Weber \& Davis (1967)-wind model, empirical spindown timescales (e.g. Skumanich 1972) imply a linear relationship between the stellar magnetic flux and the rotation rate, but sub-linear values cannot be ruled out either, owing to the impact of non-uniform surface magnetic field distributions (Holzwarth \& Jardine 2005). In contrast, Saar (2001) suggests a super-linear dependence, with a power-law index of $n_{\Phi}=1.2$, based on polarimetric observations of $\mathrm{K}$ and $\mathrm{M}$ dwarfs. Depending on the underlying stellar sample, higher values may be possible. Excluding rapid rotators in the saturated regime, Schrijver et al. (2003), for example, suggest the value $n_{\Phi}=2.8 \pm 0.3$.

\subsection{Solar reference case}

For our model to reproduce solar wind conditions observed at earth orbit $\left(v_{r} \simeq 400 \mathrm{~km} \mathrm{~s}^{-1}, n^{2} \simeq 14 \mathrm{~cm}^{-3}, T \simeq 2 \times 10^{5} \mathrm{~K}, B \simeq\right.$ $5 \times 10^{-5} \mathrm{G}$ ), the coronal values have to be $T_{0, \odot}=2.93 \times$ $10^{6} \mathrm{~K}, n_{0, \odot}=2.76 \times 10^{6} \mathrm{~cm}^{-3}, B_{0, \odot}=3 \mathrm{G}$, the polytropic in$\operatorname{dex} \Gamma=1.22$, and the mean molecular weight $\mu=0.5$ (cf. Weber \& Davis 1967; Sakurai 1985). The resulting mass flux is $F_{\mathrm{m}, \odot}=1.04 \times 10^{11} \mathrm{~g} \mathrm{~s}^{-1} \mathrm{sr}^{-1}$ and the solar mass loss rate $\dot{M}_{\odot}=4 \pi F_{\mathrm{m}, \odot}=1.31 \times 10^{12} \mathrm{~g} \mathrm{~s}^{-1}=2.07 \times 10^{-14} M_{\odot} \mathrm{yr}^{-1}$. With increasing distance from the Sun, the wind velocity converges to the value $v_{\infty, \odot}=443 \mathrm{~km} \mathrm{~s}^{-1}$, which yields the constant wind ram force per solid angle $\mathcal{F}_{\mathrm{w}, \odot} /(4 \pi)=4.60 \times 10^{18} \mathrm{dyn} \mathrm{sr}^{-1}$. With the solar radius $R_{\odot}=6.96 \times 10^{10} \mathrm{~cm}$, the CWRP of the Sun is $\mathcal{P}_{\odot}=9.51 \times 10^{-4} \mathrm{dyn} \mathrm{cm}^{-2}$.

The interaction with the local interstellar medium confines the expansion of the solar wind within a system of shock fronts, wherein velocities are braked down to sub-sonic values. At the distance of the heliospheric termination shock $(\approx 94 \mathrm{AU}$, Stone et al. 2005) the calculated solar wind has virtually obtained the terminal velocity $\left(437 \mathrm{~km} \mathrm{~s}^{-1}=0.987 v_{\infty, \odot}\right)$ and exerts a ram pressure $\left(p_{\mathrm{w}, \mathrm{TS}} \simeq 2 \times 10^{-12} \mathrm{dyn} \mathrm{cm}^{-2}\right.$, cf. Fig. 1$)$, which is in

\footnotetext{
${ }^{2}$ The average proton density in the slow solar wind is about $7 \mathrm{~cm}^{-3}$. Owing to the charge-neutrality of the plasma, we double this value to account for the free electrons. This is consistent with the mean molecular weight $\mu=0.5$.
} 


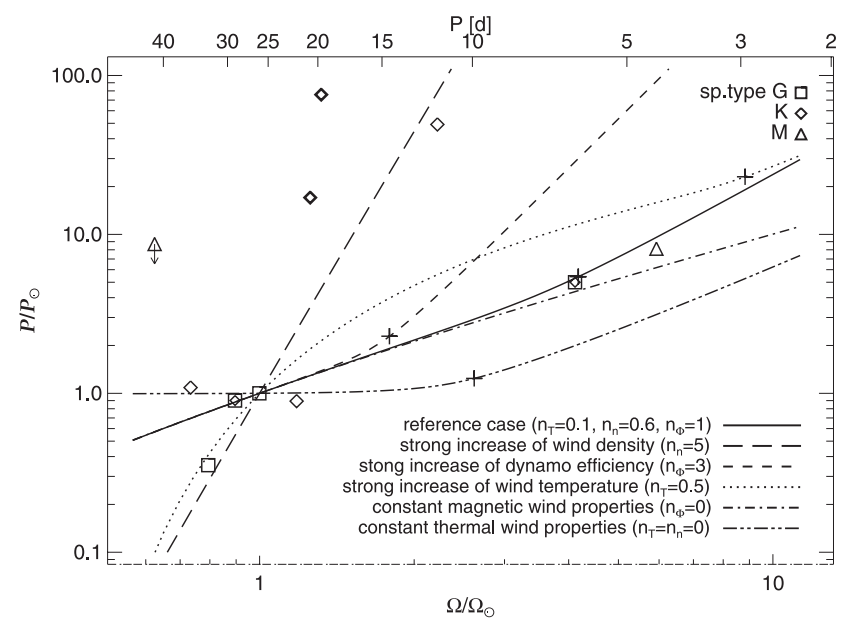

Fig. 2. Characteristic wind ram pressure (in solar units) of $1 M_{\odot}$ mainsequence stars subject to different wind scenarios (i.e. sets of power-law indices $n_{T}, n_{n}$, and $n_{\Phi}$ ). Crosses along the curves mark the transition between the regimes of slow and fast magnetic rotators, and symbols show the values inferred by Wood et al. (2005a) for main-sequence (single and binary) stars of different spectral types.

agreement with the canonical value of the pressure of the local interstellar medium (e.g. Axford \& Ip 1986).

\section{Results}

\subsection{Comparison of different wind scenarios}

An increase of thermal and/or magnetic wind parameters with the stellar rotation rate increases the CWRPs (Fig. 2). The character of the increase depends on whether the star is a slow or a fast magnetic rotator ${ }^{3}$. The winds of slow magnetic rotators are driven by thermal pressure gradients and gain energy from the enthalpy of the hot plasma. In the regime of fast magnetic rotators, stellar winds are predominantly accelerated by magnetocentrifugal driving. The outflowing plasma tries to conserve its angular momentum but is forced into faster rotation by the tension force of the bent magnetic field lines. This slingshot effect gives rise to a Poynting flux, which transfers energy from the stellar rotation into the wind.

In the regime of slow magnetic rotators, the terminal wind velocities are comparable to the solar-like surface escape velocities of main-sequence stars (Fig. 3). Except in the case of high wind temperatures, the CWRPs only depend on the mass flux, which scales almost linearly with the wind density. Higher wind temperatures entail higher mass fluxes and CWRPs, yet the increase is limited by the basic requirement of subsonic coronal flow velocities. The terminal wind velocities of fast magnetic rotators are larger than the solar value. The mass flux, however, is still determined by the thermal wind parameters. Owing to its intrinsic dependence on the stellar rotation, the dominant magneto-centrifugal driving causes an increase of the terminal wind velocity and CWRP with the stellar rotation rate even if the wind parameters are constant. The transition between the slow and the fast magnetic rotator regimes depends on the relative

${ }^{3}$ We follow the terminology of Belcher \& MacGregor (1976) and consider stars to be fast magnetic rotators, if the Michel velocity, which quantifies the impact of magnetic fields on the wind acceleration (Michel 1969), is larger than the terminal wind velocity determined in the absence of magneto-rotational effects (cf. Nerney 1980; Lamers \& Cassinelli 1999).

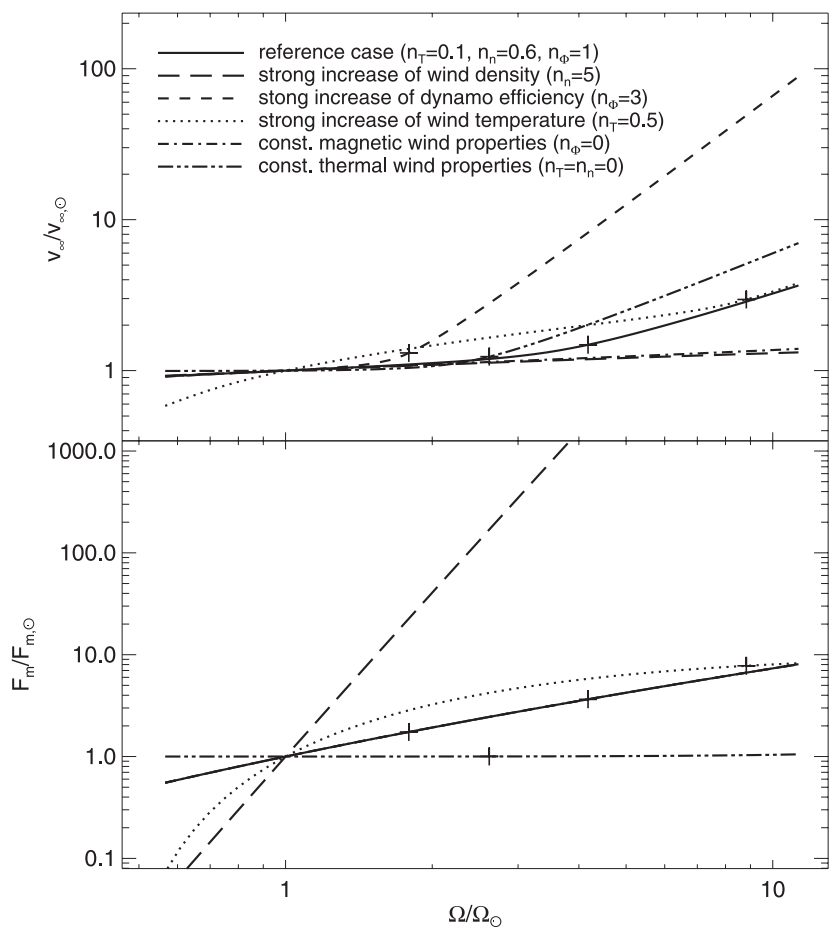

Fig. 3. Terminal velocities (top) and mass fluxes (bottom) of winds of $1 M_{\odot}$ main-sequence stars subject to different wind scenarios. In the cases of constant $\left(n_{\Phi}=0\right.$, dashed dotted $)$ and strongly increasing $\left(n_{\Phi}=3\right.$, short dashed $)$ dynamo efficiencies the mass fluxes are identical with those of the reference case (solid), since they are based on the same thermal wind parameters. However, the individual transitions between the slow and fast magnetic rotator regimes (crosses) take place at different rotation rates.

Table 1. Properties of cool main-sequence stars with observed mass loss rates, taken from Wood et al. (2005a,b).

\begin{tabular}{cccccc}
\hline \hline Star & Sp.type & $P[\mathrm{~d}]$ & $\dot{M}\left[\dot{M}_{\odot}\right]$ & $\log L_{\mathrm{X}}$ & $A\left[\mathrm{~A}_{\odot}\right]$ \\
\hline Prox Cen & M5.5 & 41.6 & $<0.2$ & 27.22 & 0.023 \\
$\alpha$ Cen & G2 / K0 & $29 / 43$ & 2 & 27.70 & 2.22 \\
$\epsilon$ Eri & K2 & 11.7 & 30 & 28.32 & 0.61 \\
61 Cyg A & K5 & 35.4 & 0.5 & 27.45 & 0.46 \\
$\epsilon$ Ind & K4.5 & 22 & 0.5 & 27.39 & 0.56 \\
36 Oph & K1 / K1 & $20.7 / 22.9$ & 15 & 28.34 & 0.88 \\
EV Lac & M3.5 & 4.38 & 1 & 28.99 & 0.123 \\
70 Oph & K0 / K5 & $19.7 / 22.9$ & 100 & 28.49 & 1.32 \\
$\xi$ Boo & G8 / K4 & $6.31 / 11.94^{a}$ & 5 & 28.90 & 1.0 \\
61 Vir & G5 & $32.7^{b}$ & 0.3 & 26.87 & 1.0 \\
\hline Sun & G2 & 26 & 1 & 27.30 & 1 \\
\hline
\end{tabular}

${ }^{a}$ From Donahue et al. (1996); ${ }^{b}$ from Noyes et al. (1984).

contribution of thermal and magneto-centrifugal driving to the overall acceleration of the wind. The higher the dynamo efficiency, $n_{\Phi}$, the stronger the magneto-centrifugal driving, and the lower the rotation rate of the transition between the two regimes. Higher wind temperatures increase the thermal driving and shift the transition to higher rotation rates.

We have compared self-consistently determined CWRPs of different wind scenarios with the observational constraints (Table 1). At present, the data set for main-sequence stars consists of three groups: five slowly rotating targets with solar-like CWRPs (Sun, $\alpha$ Cen, $\epsilon$ Ind, 61 Cyg A, 61 Vir), three moderately rotating targets of spectral type $\mathrm{K}$ with CWRPs more than ten times the solar value ( $\epsilon$ Eri, $36 \mathrm{Oph}, 70 \mathrm{Oph}$ ), and two rapidly 
rotating targets ( $\xi$ Boo, EV Lac) with 5-10 times larger CWRPs than the Sun; the value for Proxima Cen is an upper limit. We first focus on the slowly and rapidly rotating targets and consider the group of moderately rotating K dwarfs in Sect. 3.2.

Assuming a linear dynamo efficiency, $n_{\Phi}=1$, different thermal wind scenarios are (within the given observational error margins) consistent with the constraints set by the slowly and rapidly rotating stars (Fig. 2). Rotation-independent thermal wind properties appear less likely, since the resulting constant CWRPs at small rotation rates are inconsistent with the general trend of CWRPs increasing with stellar rotation. Good agreement results from moderately increasing thermal wind parameters, here $n_{T}=0.1$ and $n_{n}=0.6$ (reference case), but the empirical constraints do not conclusively exclude the scenario either, in which the wind temperature follows the high-temperature coronal plasma component (i.e. $n_{T}=0.5$ ). In the latter scenario, the strong increase of CWRPs at low rotation rates, which then becomes flatter for higher rotation rates, appears promising to account for the high values of the group of K dwarfs. But we find that even for $n_{T} \gg 0.5$ the CWRPs are not sufficiently high. The only scenario consistent with these targets is based on a very strong increase of the coronal density with the stellar rotation rate. For stars close to the X-ray saturation limit, rotating about ten times faster than the Sun, values of $n_{n} \gtrsim 5$ imply very high wind densities and CWRPs which are inconsistent with the lower values inferred for the rapidly rotating targets. The scenario of wind ram pressures scaling exclusively with the wind density corresponds to the approach of Wood et al. (2002).

Retaining the thermal power-law indices $n_{T}=0.1$ and $n_{n}=$ 0.6 , the theoretical CWRPs are consistent with the constraints set by the slowly and rapidly rotating targets for dynamo efficiencies $0<n_{\Phi} \lesssim 1.5$. For $n_{\Phi}<1$ all stars considered here are slow magnetic rotators, whereas for super-linear dynamo efficiencies the two rapidly rotating targets, $\xi$ Boo and EV Lac, are in the fast magnetic rotator regime. A rather high but previously suggested dynamo efficiency of $n_{\Phi} \sim 3$ locates the transition between slow and fast magnetic rotators at rotation rates similar to those of the $\mathrm{K}$ dwarf group. Yet due to their moderate rotation, the magnetocentrifugal driving is too inefficient and cannot produce CWRPs sufficiently high to match the empirical values.

None of the scenarios above is capable to account for all of the empirical constraints, and all but one scenario are incapable to match the high CWRPs of the group of moderately rotating $\mathrm{K}$ dwarfs. With highest values occurring at intermediate rotation rates, it is unlikely that the wind ram pressures of the three groups can be described using simple power-law relations for the thermal and magnetic wind properties. The group of moderately rotating $\mathrm{K}$ dwarfs shows high $\mathrm{CWRP}$, which would require a sudden change of wind properties with the stellar rotation rate. But assuming a continuous dependency makes it difficult to yield lower CWRPs at higher stellar rotation rates, as suggested by $\xi$ Boo and EV Lac. In contrast, a weak dependence on the stellar rotation rate enables to match the values of the rapidly rotating targets, but cannot account for the high CWRPs of the K dwarf group. This suggests that either the group of rapidly rotating targets or the group of moderately rotating $\mathrm{K} \mathrm{dwarfs}$ are peculiar in terms of stellar wind properties.

\subsection{The group of moderately rotating $K$ dwarfs}

In the framework of our wind model with rotation-dependent thermal and magnetic wind parameters, the CWRPs of the three moderately rotating targets $36 \mathrm{Oph}\left(\mathcal{P} / \mathcal{P}_{\odot}=17\right), 70 \mathrm{Oph}$ $\left(\mathcal{P} / \mathcal{P}_{\odot}=75\right)$, and $\epsilon$ Eri $\left(\mathcal{P} / \mathcal{P}_{\odot}=49\right)$, could only be accounted

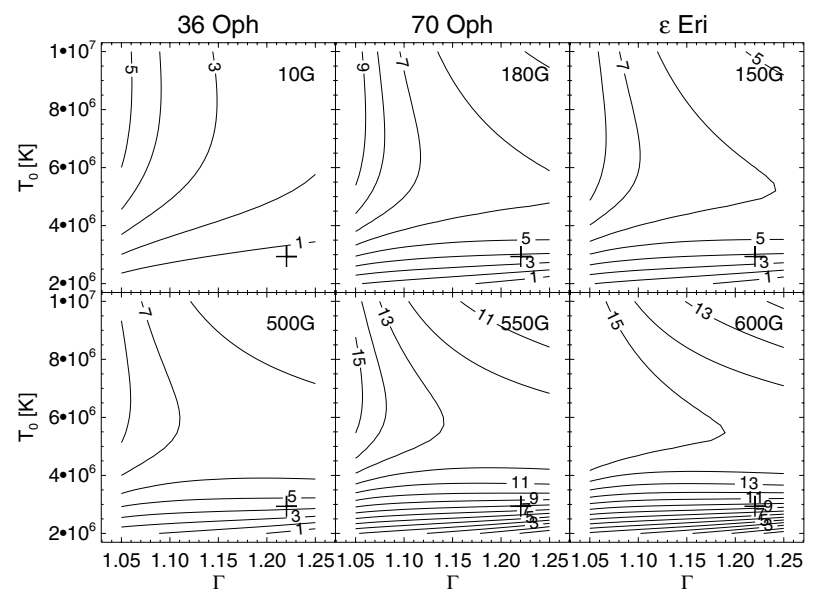

Fig. 4. Theoretical characteristic wind ram pressures (in solar units) of the moderately rotating $\mathrm{K}$ dwarf targets as function of the wind temperature, $T_{0}$, at the coronal base and the polytropic index, $\Gamma$; the cross marks solar reference values. The magnetic field strengths, $B_{0}$ (labels), are lower and upper limits of observed field strength ranges. The stellar model parameters are for $36 \mathrm{Oph}: M=0.63 M_{\odot}, r_{0}=0.76 R_{\odot}, \Omega=$ $3.5 \times 10^{-6} \mathrm{~s}^{-1} ; 70 \mathrm{Oph}: M=0.8 M_{\odot}, r_{0}=0.94 R_{\odot}, \Omega=3.7 \times 10^{-6} \mathrm{~s}^{-1}$; $\epsilon$ Eri: $M=0.73 M_{\odot}, r_{0}=0.86 R_{\odot}, \Omega=6.2 \times 10^{-6} \mathrm{~s}^{-1}$. The wind density at the reference level is $n_{0}=2.76 \times 10^{6} \mathrm{~cm}^{-3}$ and the mean molecular weight $\mu=0.5$.

for by a strong increase of wind densities with stellar rotation rate. For otherwise solar (i.e. reference) wind parameters, the wind densities required to produce the empirical CWRPs are about 25 times ( $36 \mathrm{Oph}), 82$ times ( $70 \mathrm{Oph}$ ), and 65 times the solar value. Yet, according to analyses of recent X-ray observations (Wood \& Linsky 2006), the coronae of the three targets are solarlike as far as densities are concerned. We investigate what other wind conditions may cause high CWRPs, and whether these are consistent with observations.

Regarding their rather slow rotation, the three targets have unexpectedly high surface-averaged magnetic field strengths: (0)-500 G for 36 Oph (Marcy 1984; Saar \& Osten 1997), 180-550 G for 70 Oph (Robinson et al. 1980; Marcy 1984), and 150-600 G for $\epsilon$ Eri (Marcy 1984; Valenti et al. 1995; Rueedi et al. 1997). The lower values are considered to be more reliable, but owing to magnetic filling factors smaller one peak field strengths may locally reach $1-3 \mathrm{kG}$. We determine CWRPs for the three targets as functions of both the wind temperature at the reference level and the polytropic index. The wind density at the reference level is taken to be $n_{0}=2.76 \times 10^{6} \mathrm{~cm}^{-3}$, whereas individual stellar rotation rates, radii, and masses are taken from Table 1, Wood et al. (2005b), and the approximation $M \propto R^{5 / 4}$ (cf. Sect. 2.1), respectively. The calculations are carried out for the lower and upper limits of each magnetic field strength range given above (Fig. 4). For drastic thermal wind conditions, like base temperatures $T_{0} \lesssim 10^{7} \mathrm{~K}$ and $\Gamma \gtrsim 1$ (implying almost isothermal outflows), CWRPs increase to about 5-15 times the solar value. Despite the large observational uncertainties of a factor of two (Wood et al. 2002), the resulting CWRPs are insufficient to achieve conclusive agreements with the empirical values.

The Chandra observations of Wood \& Linsky (2006) show abundance anomalies for some of the $\mathrm{K}$ dwarf targets in the form of a FIP effect, that is the relative abundances (with respect to photospheric values) of elements with low first ionisation potentials are enhanced compared to high-FIP elements. The element abundances determine the mean molecular weight 


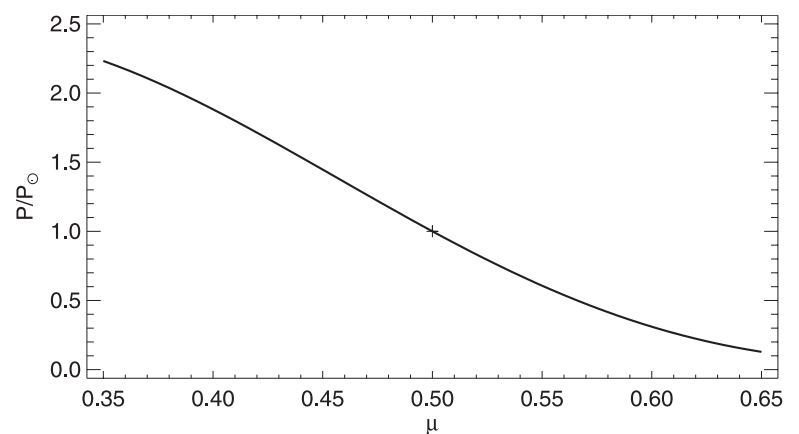

Fig. 5. Dependence of the characteristic wind ram pressure (in solar units) on the mean molecular weight, assuming solar reference values $\left(M=1 M_{\odot}, \Omega=2.8 \times 10^{-6} \mathrm{~s}^{-1}, r_{0}=1.1 R_{\odot}, B_{0}=3 \mathrm{G}, T_{0}=\right.$ $\left.2.93 \times 10^{6} \mathrm{~K}, n_{0}=2.76 \times 10^{6} \mathrm{~cm}^{-3}, \Gamma=1.22\right)$.

of the coronal plasma, and could thus have an influence on the wind acceleration mechanisms and mass loss rate. Assuming a fully ionised plasma, solar photospheric abundances (Grevesse $\&$ Sauval 1998) yield $\mu_{\odot, \mathrm{ph}} \approx 0.61$. In the solar wind, elements with FIP $<10 \mathrm{eV}$ are about 4.5 times overabundant and elements with $10 \mathrm{eV}<$ FIP $<11.5 \mathrm{eV}$, in particular $\mathrm{C}$ and $\mathrm{S}$, about two times overabundant (Geiss 1998; Raymond 1999); in the slow wind, $\mathrm{He}(\mathrm{FIP} \sim 25 \mathrm{eV})$ is about two times underabundant (Geiss 1998). These abundance differences result in $\mu_{\odot \text {, wind }} \approx 0.57$. Wood \& Linsky (2006) determined the relative abundances of the major constituents of the $\mathrm{K} \mathrm{dwarf}$ coronae. If we assume that in stellar winds He is generally underabundant, the mean molecular weights are $\mu_{36 \mathrm{Oph}(\mathrm{A} / \mathrm{B})} \approx 0.53, \mu_{70 \mathrm{Oph}(\mathrm{A})} \approx$ $0.58, \mu_{70 \mathrm{Oph}(\mathrm{B})} \approx 0.54$, and $\mu_{\epsilon \text { Eri }} \approx 0.54$, that is $\lesssim 10 \%$ smaller than the solar value. However, the dependence of the CWRP on the mean molecular weight is small (Fig. 5), and this effect therefore rather marginal.

In summary, high magnetic field strengths, high wind temperatures, high heating rates and low mean molecular weights do, in principle, increase the CWRPs of cool stars. Yet, if the energy flux into open and closed magnetic field structures is similar, then we expect that the extreme coronal conditions required to yield CWRPs nearly two orders of magnitude larger than the Sun would imprint distinctive signatures on stellar X-ray properties. Since these signatures are not discernible in the case of the moderately rotating $\mathrm{K}$ dwarfs, their high CWRPs are peculiar.

\subsection{Dependence on spectral type}

For comparable wind parameters and rotation rates, the CWRPs of lower-mass main-sequence stars are higher than for solarmass stars. Over the mass range $0.2-1.2 M_{\odot}$, the difference is typically smaller than about half an order of magnitude (Fig. 6). This holds for both the slow and the fast magnetic rotator regime, so it is unrelated to the higher magnetic field strengths for lower mass stars, adapted to retain the total magnetic flux (cf. Sect. 2.4). The increase is caused by the scaling of $\mathcal{P}$ with the inverse surface area (i.e. $\propto R^{-2}$ ). The mass fluxes (per solid angle) of lower-mass stars are, in fact, significantly smaller than the solar value. In the case of the reference wind scenario, the CWRPs and mass loss rates follow approximately broken powerlaws (Table 2), whose parameters reflect the result above. For $\mathrm{K}$ dwarfs with masses $\sim 0.7 M_{\odot}$, the increase of the CWRP is clearly insufficient to account for the high observed values of the peculiar group of moderately rotating targets.

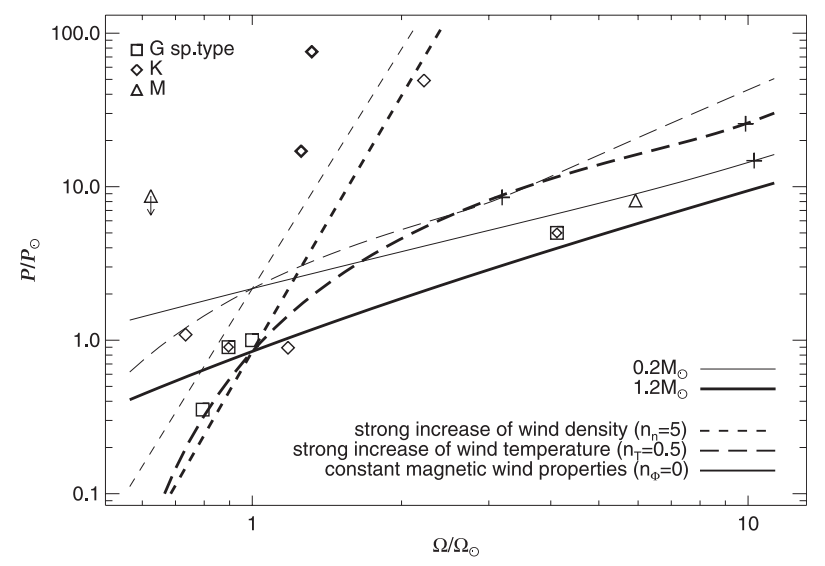

Fig. 6. Characteristic wind ram pressures of $1.2 M_{\odot}$ (thick lines) and $0.2 M_{\odot}$ (thin lines) main-sequence stars, subject to different wind scenarios. Crosses mark the transition between the slow and the fast magnetic rotator regimes, symbols show the empirical values given by Wood et al. (2005a).

\subsection{Inclination effects}

The wind ram pressure of magnetised winds is intrinsically latitude-dependent, since plasma emanating along open magnetic field lines at high latitudes experience a weaker magnetocentrifugal driving than outflows in the equatorial plane. Inferred mass loss rates based on the assumption of spherical symmetric outflows may misestimate the actual value if the inclination between the line-of-sight and the rotation axis is not taken into account. We analyse the impact of this effect by determining latitude-dependent CWRPs. The applied model is an extension of the Weber \& Davis-formalism to non-equatorial latitudes, assuming that the poloidal magnetic field component is radial, so that the spiralling field lines are located on conii with constant opening angles, whose tips are located in the centre of the star (Holzwarth 2005).

Disregarding possible anisotropies caused by closed coronal magnetic field structures, the thermally driven winds of slow magnetic rotators are virtually spherical symmetric and inferred CWRPs independent of inclination effects. In contrast, in the regime of fast magnetic rotators, possible CWRPs range over up to an order of magnitude, depending on the underlying wind scenario (Fig. 7). Small inclinations between the rotation axis and the line-of-sight typically imply smaller values. Considering the larger surface area at lower latitudes, the expectation values are closer to the higher CWRPs determined in the equatorial plane.

The present analysis is based on uniform magnetic field distributions. Yet observations of rapidly rotating stars frequently show non-uniform surface brightness and magnetic field distributions in the form of spot concentrations at high latitudes (e.g. Strassmeier 2002, and references therein), which alter the latitudinal variation of the wind structure. Magnetised winds of rapidly rotating stars furthermore show a collimation of open magnetic field lines toward the rotation axis, which causes an additional latitude-dependence of the wind structure (e.g. Tsinganos \& Bogovalov 2000). Such anisotropies increase the range of possible CWRPs and the uncertainty of inferred values.

\subsection{Rotation-activity-relationship}

The activity level of cool stars is typically quantified through their coronal X-ray emission; Wood et al. (2002, 2005a) correlate the inferred mass loss rates with observed X-ray fluxes. 
Table 2. Power-law approximations for the CWRP, $\mathcal{P}=\overline{\mathcal{P}}\left(\Omega / \Omega_{\odot}\right)^{n_{\mathcal{P}}}$, the mass flux (per solid angle), $F_{\mathrm{m}}=\dot{M} /(4 \pi)=\bar{F}_{\mathrm{m}}\left(\Omega / \Omega_{\odot}\right)^{n_{F}}$, and the terminal wind velocity, $v_{\infty}=\bar{v}_{\infty}\left(\Omega / \Omega_{\odot}\right)^{n_{v}}$, of main-sequence stars, assuming a wind scenario with $n_{T}=0.1, n_{n}=0.6$, and $n_{\Phi}=1$. The transition between the slow and the fast magnetic rotator regime, SMR and FMR, respectively, takes place at $\Omega_{\mathrm{s} / \mathrm{f}}$.

\begin{tabular}{|c|c|c|c|c|c|c|c|c|c|c|c|c|c|}
\hline \multirow{2}{*}{$\begin{array}{c}M \\
{\left[M_{\odot}\right]}\end{array}$} & \multirow{2}{*}{$\begin{array}{l}\Omega_{\mathrm{s} / \mathrm{f}} \\
{\left[\Omega_{\odot}\right]} \\
\end{array}$} & \multicolumn{6}{|c|}{ SMR $\left(\Omega<\Omega_{\mathrm{s} / \mathrm{f}}\right)$} & \multicolumn{6}{|c|}{ FMR $\left(\Omega>\Omega_{\mathrm{s} / \mathrm{f}}\right)$} \\
\hline & & $\overline{\mathcal{P}}\left[\mathcal{P}_{\odot}\right]$ & $n_{\mathcal{P}}$ & $\bar{F}_{\mathrm{m}}\left[\mathrm{F}_{\mathrm{m}, \odot}\right]$ & $n_{F}$ & $\bar{v}_{\infty}\left[v_{\infty, \odot}\right]$ & $n_{v}$ & $\overline{\mathcal{P}}\left[\mathcal{P}_{\odot}\right]$ & $n_{\mathcal{P}}$ & $\bar{F}_{\mathrm{m}}\left[\mathrm{F}_{\mathrm{m}, \odot}\right]$ & $n_{F}$ & $\bar{v}_{\infty}\left[v_{\infty, \odot}\right]$ & $n_{v}$ \\
\hline 1.2 & 4.39 & 0.83 & 1.21 & 1.15 & 0.99 & 0.97 & 0.21 & 0.40 & 1.73 & 1.41 & 0.82 & 0.38 & 0.90 \\
\hline 1.0 & 4.18 & 0.99 & 1.14 & 0.99 & 0.94 & 1.00 & 0.20 & 0.45 & 1.73 & 1.18 & 0.79 & 0.38 & 0.93 \\
\hline 0.8 & 3.90 & 1.18 & 1.08 & 0.80 & 0.89 & 1.04 & 0.19 & 0.52 & 1.72 & 0.92 & 0.76 & 0.39 & 0.95 \\
\hline 0.6 & 3.54 & 1.43 & 1.01 & 0.58 & 0.84 & 1.09 & 0.17 & 0.61 & 1.72 & 0.66 & 0.73 & 0.41 & 0.99 \\
\hline 0.4 & 3.05 & 1.75 & 0.94 & 0.35 & 0.78 & 1.14 & 0.16 & 0.77 & 1.72 & 0.38 & 0.70 & 0.46 & 1.02 \\
\hline 0.2 & 2.30 & 2.17 & 0.85 & 0.13 & 0.71 & 1.23 & 0.14 & 1.10 & 1.72 & 0.14 & 0.65 & 0.59 & 1.07 \\
\hline
\end{tabular}

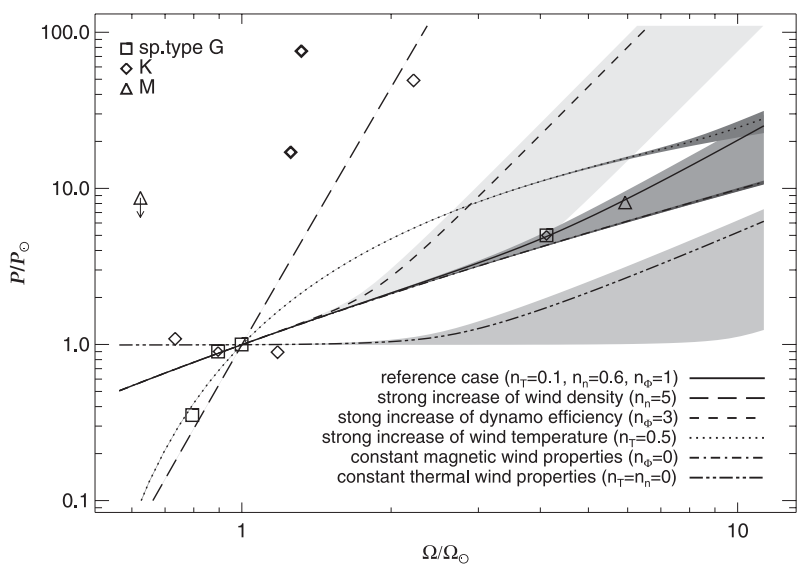

Fig. 7. Characteristic wind ram pressures of outflowing plasma with different inclinations to the stellar rotation axis. Based on uniform surface magnetic field distributions, higher (lower) values within a shaded region are associated with equatorial (high) latitudes. The lines show surface-averaged mean values, that is the values one would statistically expect if the inclination is unknown. Symbols indicate the values and spectral types of the empirical data of Wood et al. (2005a).

Table 3. Coefficients and power-law indices of empirical rotationactivity-relationships. The mass dependence has been determined by fitting power laws to the characteristic values of each mass bin in the data set given by Pizzolato et al. (2003).

\begin{tabular}{ccc}
\hline \hline $\lg \bar{F}_{X}\left[\mathrm{erg} \mathrm{s}^{-1} \mathrm{~cm}^{-2}\right]$ & $n_{x}$ & Sample \\
\hline $15.51+0.623\left(\frac{M}{M_{\odot}}\right)-2 \lg \left(\frac{R}{R_{\odot}}\right)$ & 2 & $259 \mathrm{FG} \mathrm{KM} \mathrm{stars}^{a}$ \\
$19.25-2 \lg \left(\frac{R}{R_{\odot}}\right)$ & 2.64 & $9 \mathrm{G} \mathrm{stars}{ }^{b}$ \\
17.88 & 2.4 & $19 \mathrm{~F} \mathrm{G} \mathrm{stars}^{c}$ \\
\hline
\end{tabular}

${ }^{a}$ Pizzolato et al. (2003); ${ }^{b}$ Güdel et al. (1997); ${ }^{c}$ Wood et al. (2005b).

For cool stars with rotation periods longer than about two days, $\mathrm{X}$-ray fluxes increase with the stellar rotation rate, following (on average) a power-law relation,

$F_{\mathrm{X}}=\bar{F}_{\mathrm{X}} \Omega^{n_{\mathrm{X}}}$.

The empirical values $\bar{F}$ and $n_{\mathrm{X}}$ depend on the spectral type and size of the underlying stellar sample. We demonstrate the impact of these quantities on the mapping of our theoretical

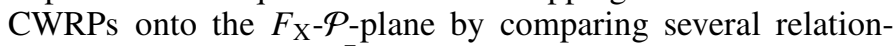
ships (Table 3 ). Larger $\bar{F}_{\mathrm{X}}$-values shift the curves of theoretical CWRPs to higher coronal X-ray fluxes, whereas smaller powerlaw indices $n_{\mathrm{X}}$ cause steeper curves, since $\mathrm{d} \ln \mathcal{P} / \mathrm{d} \ln F_{\mathrm{X}}=$ $(\mathrm{d} \ln \mathcal{P} / \mathrm{d} \ln \Omega) / n_{\mathrm{X}}$ (Fig. 8). The latter effect would ease the need for a strong increase of the wind density with the stellar rotation. Depending on the applied rotation-activity relation, values $n_{n} \simeq 2.2-3.1$ yield CWRPs which are in agreement with

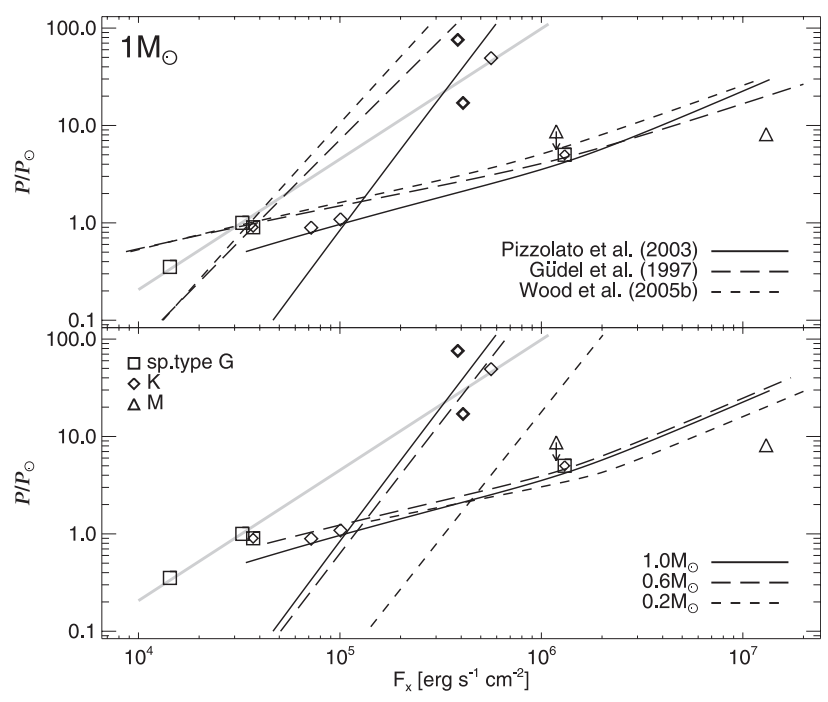

Fig. 8. Characteristic wind ram pressures (in solar units) as function of the stellar X-ray flux, assuming the reference $\left(n_{T}=0.1, n_{n}=0.6, n_{\Phi}=\right.$ $1)$ and the high wind density $\left(n_{T}=0.1, n_{n}=5, n_{\Phi}=1\right.$, steep curves $)$ scenarios. Different empirical rotation-activity-relations result in different locations and slopes of the curves for a $1 M_{\odot}$ star (top). Using the rotation-activity relationship based on data by Pizzolato et al. (2003), the dependence on spectral type (i.e. stellar mass) shifts the values of lower-mass stars to higher X-ray fluxes (bottom). The gray line marks the X-ray flux-mass loss-relation, $\dot{M} \propto F_{\mathrm{X}}^{1.34}$, suggested by Wood et al. (2005a).

the moderately rotating $\mathrm{K}$ dwarfs and the power-law relationship suggested by Wood et al. (2005a). Yet the offsets caused by different $\bar{F}_{\mathrm{X}}$ result in inconsistencies between theoretical and empirical CWRPs.

The range of possible locations and slopes of CWRP curves resulting from different empirical rotation-activity relations make it difficult to associate inferred mass loss rates with a systemic change of stellar wind parameters. The rotationactivity relationships are statistical relations based on a (relative) large number of stars. For individual objects, deviations from the mean value can be significant and represent an additional source of uncertainty in the analysis of stellar wind ram pressures. Furthermore, X-ray fluxes of cool stars are typically not constant, but may vary in the course of an activity cycle, in the case of the Sun by an order of magnitude.

\subsection{Stellar mass loss rates}

Following Eqs. (5) and (6), a match between theoretical and empirical CWRPs implies the relation

$\left(\frac{\dot{M}}{\dot{M}_{\odot}}\right)_{\mathrm{W}} \stackrel{!}{=} \frac{\dot{M}}{\dot{M}_{\odot}} \frac{v_{\infty}}{v_{\infty, \odot}}$ 


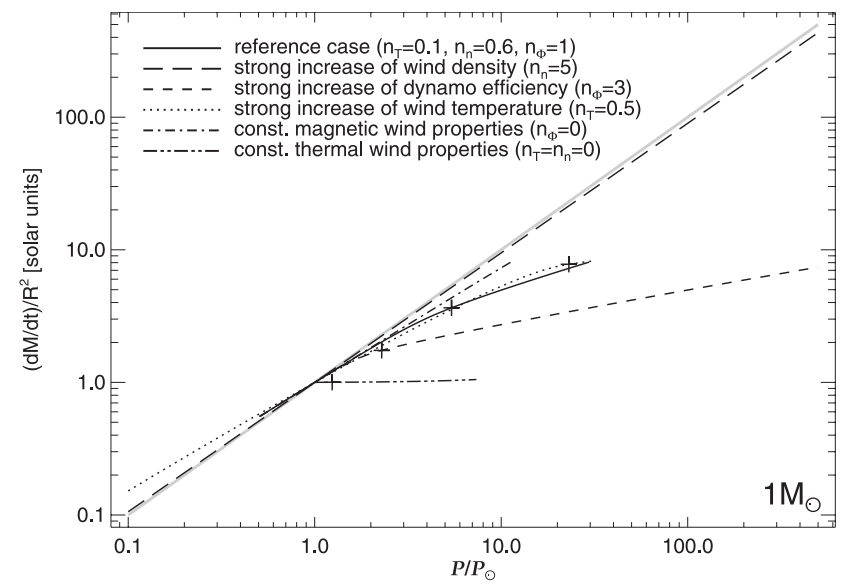

Fig. 9. Self-consistent mass loss rates per surface area, $\dot{M} / R^{2}$ (in solar units), as a function of the relative characteristic wind ram pressure, $\mathcal{P} / \mathcal{P}_{\odot}$. Subject to the condition $v_{\infty}=v_{\infty, \odot}$, the latter quantity is equivalent to the empirical mass loss rates per surface area given by Wood et al. Crosses mark the transition between the regimes of slow and fast magnetic rotators; the gray line indicates identity between theoretical and observed mass loss rates.

If the terminal wind velocity is different from the solar value, then the self-consistently determined mass loss rate, $\dot{M}$, is different from the value, $\dot{M}_{\mathrm{W}}$, derived by Wood et al. (2002), since the latter is based on the assumption of a unique solar-like wind velocity. A comparison between theoretical and empirical stellar mass loss rates is shown in Fig. 9 for a $1 M_{\odot}$ star subject to different wind scenarios. Note that, following Eq. (6), the relative CWRPs on the abscissa are equivalent to the empirical mass loss rates per surface area of Wood et al. (2002). For thermally driven winds of slow magnetic rotators, there is an almost one-to-one correspondence between self-consistent and observed mass loss rates, with the possible exception of very hot stellar winds. In contrast, in the regime of fast magnetic rotators, the terminal velocities of magneto-centrifugally driven winds are faster than in the solar case (see Fig. 3), and the self-consistent mass loss rates thus smaller than those determined following the approach of Wood et al. In all but one of our wind scenarios the theoretical mass loss rates do not exceed about ten times the solar value. Only if the wind ram pressure scales exclusively with the wind density are theoretical and empirical mass loss rates similar throughout. In terms of this scenario, all stars are slow magnetic rotators with solar-like winds, whose CWRPs scale with the mass flux. The results for lower-mass stars are qualitatively the same, with stellar mass loss rates (per surface area) not exceeding about ten times the solar value either. We conjecture that disregarding the importance of the (terminal) wind velocity on the CWRP may lead to over-estimations of the mass loss rates of rapidly rotating stars.

\section{Discussion}

The aim of our investigation was to develop a picture of the wind ram pressures and mass loss rates of cool main-sequence stars. Rather than analysing individual stars in detail, we have used the set of observationally determined values to constrain possible wind scenarios. In view of large observational uncertainties and the small and heterogeneous sample of stars, we did not attempt to fit theoretical and empirical values, but used the latter as guidance only. The power-law ansatz for the dependence of the thermal and magnetic wind parameters on the stellar rotation rate is motivated and supported by previous investigations. Nevertheless, with highest currently observed CWRPs occurring at moderate rotation rates, it is not possible to find a consistent agreement between a theoretical wind scenario and all empirical values. This problem raises the question whether the increase of CWRPs is characterised by the group of moderately rotating $\mathrm{K}$ dwarfs or by the group of rapidly rotating targets.

\subsection{The K dwarf puzzle}

The high CWRPs of the K dwarfs $\epsilon$ Eri, 70 Oph, and 36 Oph cannot be explained in the framework of solar-like magnetised winds. The temperatures, densities, and heating rates required to raise the values are inconsistent with the X-ray observations of Wood \& Linsky (2006). Strong magnetic fields could account for their CWRPs, but the observed magnetic flux densities of these $\mathrm{K}$ dwarfs, albeit being unexpectedly high, are insufficient. On the observational side, inaccurate assumptions about the ambient ISM or the difficult fitting procedure of simulated and observed astrospheric absorption profiles may cause overestimations of the empirical mass loss rates. On the theoretical side, our model may miss out on additional wind acceleration or energy transfer mechanisms, which could cause higher terminal velocities. However, we expect any effect capable of increasing the CWRP up to 80 times the solar value to render stellar winds non-solar and to imprint discernible signatures on the coronal X-ray properties of the star. Based on the available results, we consider the high CWRPs of the group of K dwarfs to be peculiar and uncharacteristic for the winds of cool main-sequence stars.

\subsection{CWRP of cool main-sequence stars}

Assuming that the increase of the CWRP of main-sequence stars is characterised by the rapidly rotating targets $\xi$ Boo and EV Lac, we argue in favour of stellar winds whose thermal and magnetic properties increase moderately with the stellar rotation rate like, for example, the wind scenario with $n_{T}=0.1, n_{n}=0.6$, and $n_{\Phi}=1$. This set of parameters yields CWRPs in good agreement with the values of the slow and rapidly rotating stars, and is based on previous, independent investigations on the increase of thermal and magnetic coronal properties (e.g. Ivanova \& Taam 2003; Favata \& Micela 2003). However, the large uncertainties of the empirical mass loss rates also allow for wind scenarios with parameters covering some range around these reference values to be in agreement with the empirical constraints.

Our theoretical results indicate that for a given rotation rate and similar wind conditions, the CWRPs of cool stars increase toward later spectral types. The increase is due to the shrinking surface area; the mass loss rates of lower mass stars are actually predicted to decrease. The increase of the CWRP depends to some extent on our approximation of stellar radii, $R \propto M^{0.8}$, on the lower main-sequence, and we thus expect it to be diluted by the dependence on the actual radii and mass loss rates of individual stars. Since over the mass range of cool stars the difference is smaller than the observational uncertainties, a structuring of CWRPs according to stellar spectral type will be hardly discernible. The situation worsens when the analysis is carried out in terms of the coronal X-ray flux instead of the rotation, since the statistical character of a rotation-activity relationship as well as intrinsic (e.g. cyclic) variations of the $\mathrm{X}$-ray emission add to the scatter of empirical values. In particular in the regime of fast magnetic rotators, we expect large scatter 
of observed CWRPs around the mean values, caused by different inclinations between stellar rotation axes and lines-of-sight. The collimation of open magnetic field lines toward the stellar rotation axis and non-uniform surface distributions of magnetic flux make the wind ram pressure of rapidly rotating stars intrinsically latitude-dependent (e.g. Tsinganos \& Bogovalov 2000; Holzwarth 2005). Since astrospheres represent the impact of stellar winds averaged over longitude and long (possibly decadal) timescales, small-scale and intermittent variations will be smeared out. Yet the typical concentration of magnetic flux at polar latitudes (Strassmeier 2002) as well as the collimation of magnetic field lines may cause a gradient in the wind ram pressure between equatorial and polar latitudes. A more detailed analysis is required to quantify the possible impact of these latitude-dependent effects. Unfortunately, the inclination of a star is seldom known, so that these effects can hardly be verified observationally. But it is advisable to take them into account as a possible source of scatter in the regime of fast magnetic rotators.

In their analysis of the X-ray properties of the moderately rotating K dwarfs, Wood \& Linsky (2006) find a possible connection between the strength of their winds and coronal abundance anomalies. Our investigation confirms a dependence of the CWRP on the FIP effect. The influence on the mean molecular weight is small and the associated impact on the CWRP well below the observational accuracy, but the trend confirms the finding of Wood \& Linsky, that a strong FIP effect implies a weaker stellar wind. Yet we caution that our polytropic, hydrodynamic ansatz does probably not allow for an adequate analysis of this question, and that a dedicated investigation is required to clarify this point.

\subsection{Stellar mass loss rates}

Disregarding the terminal wind velocity in the analysis of CWRPs can cause misestimations of stellar mass loss rates. For fast magnetic rotators, the self-consistently determined mass loss rates are typically smaller than the values based on the presumption of solar-like winds, and do not exceed about ten times the solar value, which is in agreement with upper limits based on observations of dMe stars (Lim \& White 1996; van den Oord \& Doyle 1997).

Since the terminal wind velocities of fast magnetic rotators are higher than solar-like values, mass loss rates more than an order of magnitude higher than the actual values may be deduced. Unfortunately, the wind velocities of cool stars are observationally not constrained. But due to the additional magnetocentrifugal driving mechanism a similarity with surface escape velocities, like in the case of thermal winds of slow rotators, cannot be expected per ser. Very fast terminal wind velocities though require magnetic fields of several kilo-Gauss, high filling factors, and high dynamo efficiencies, which are observationally not confirmed and in contrast with current theories on dynamo operation/saturation and on the rotational evolution of cool stars. Since the angular momentum loss associated with high magnetic fluxes brakes the stellar rotation too efficiently, the observations of rapidly rotating zero-age-main-sequence stars would be difficult to explain (Holzwarth \& Jardine 2005, and below). We consider wind scenarios based on high dynamo efficiencies to be marginal, albeit respective values have been previously suggested (Schrijver et al. 2003, though focusing on a different aspect of stellar activity). The predicted range of dynamo efficiencies is consistent with the observationally determined, super-linear values of Saar (2001).

\subsection{Comparison of energy fluxes}

Combining our power-law approximations for the stellar mass loss rates with the rotation-activity relationship, Eq. (9), yields $\dot{M} \propto F_{\mathrm{X}}^{n_{F} / n_{\mathrm{X}}}$. With the values given in Tables 2 and 3 , the rate of increase, $n_{F} / n_{\mathrm{X}} \sim 0.5$, is significantly smaller than the value $1.34 \pm 0.18$, suggested by Wood et al. (2005a). The reason for this difference is that we assume the increase of CWRPs to be characterised through the lower values of the rapidly rotating targets, whereas Wood et al. base their fit on the high CWRP of the peculiar group of moderately rotating $\mathrm{K}$ dwarfs.

The transport of polytropic gas from the stellar surface to infinity requires the specific thermal energy (per unit mass)

$q=\frac{(\gamma-\Gamma)}{(\gamma-1)(\Gamma-1)} \frac{\mathfrak{R}}{\mu} T_{0}$

which has to be provided by the star. Thus, at the stellar surface the thermal energy flux along open magnetic fields must be

$F_{\mathrm{W}}=\frac{q \dot{M}}{4 \pi R^{2}}=\frac{q \mathcal{P}}{v_{\infty}}$

or, in the case of $1 M_{\odot}$ stars with rotation rates $\lesssim 4.2 \Omega_{\odot}$, approximately

$F_{\mathrm{W}} \approx 3.23 \times 10^{4}\left(\frac{\Omega}{\Omega_{\odot}}\right)^{1.04}\left[\frac{\mathrm{erg}}{\mathrm{s} \mathrm{cm}^{2}}\right]$,

assuming a mono-atomic gas with a ratio of specific heats $\gamma=5 / 3$. At solar rotation rate, our approximation is in agreement with the empirical values for quite sun regions, $F_{\mathrm{W}} \lesssim$ $5 \times 10^{4} \mathrm{erg} \mathrm{s}^{-1} \mathrm{~cm}^{-2}$, but an order of magnitude smaller than the value for coronal holes (Withbroe \& Noyes 1977). We note that the wind parameters we have used to gauge our model at earth orbit (cf. Sect. 2.5) are characteristic for the slow solar wind, which is expected to originate from quite sun regions, whereas coronal holes are harbouring the fast solar wind component. In comparison with the increase of the coronal X-ray flux, $F_{\mathrm{X}} \propto \Omega^{n_{\mathrm{X}}}$ with $n_{x} \gtrsim 2$, the thermal energy flux of stellar winds is predicted to increases with a much lower rate.

The coronal X-ray flux quantifies the energy input into closed magnetic field regions, whereas the wind energy flux quantifies the (thermal) energy input into wind regions. Like Wood et al. (2002), we do not expect a direct connection between the two energy fluxes. Closed magnetic field regions are mainly heated by the dissipation of magnetic energy through reconnection processes. Pevtsov et al. (2003), for example, finds a correlation between stellar X-ray luminosities and magnetic flux, $L_{\mathrm{X}} \propto \Phi^{1.15}$. A dominant magnetic heating of wind regions is unlikely, since reconnecting field lines would hamper the acceleration of coherent plasma motions to super-sonic/super-Alfvénic flow velocities. Instead, the Poynting flux presents an additional energy source for the outflowing plasma, with magnetic field lines transferring rotational energy of the star into kinetic energy without being dissipated. The different rates of increase of the X-ray flux and the wind energy flux may be due to different dependencies of the heating mechanisms on the stellar rotation rate. Correlating the stellar mass loss rate or wind energy flux with the coronal X-ray flux may eliminate the explicit dependence on the rotation rate, but it is questionable whether there is a direct physical relationship between the two quantities.

For the X-ray luminosity-magnetic flux relation of Pevtsov et al. to be consistent with empirical rotation-activity relationships (cf. Table 3 ) requires dynamo efficiencies $n_{\Phi} \sim 2$, which 
would yield CWRPs outside the errorbars of the rapidly rotating targets. Such high dynamo efficiencies have severe ramifications for the rotational evolution of cool stars, since high magnetic flux densities imply a strong magnetic braking. For instance, the observed spin-down of main-sequence stars with time, $\Omega \propto \sqrt{t}$ (Skumanich 1972), is consistent with a linear dynamo efficiency. The inconsistency between rotation-activity relations, on one side, and stellar spin-down timescales, on the other, may be caused by the former being related to the closed magnetic flux and the latter to the open magnetic flux, indicating a different dependence of the open and closed magnetic field structures on the stellar rotation rate.

\subsection{Impact on stars and planets}

The increase of the mass loss rate with stellar rotation, $\dot{M} \propto \Omega^{n_{F}}$, is according to our results $\left(n_{F} \lesssim 1\right)$ much weaker than predicted by Wood et al. (2002): $n_{F} \approx 3.3$ (cf. combination of their Eqs. (1) and (3)). For rapidly rotating stars, our mass loss rates are smaller than their predictions, whereas for slowly rotating stars both predictions are similar. We illustrate the consequences of the different rates of increase by comparing the effect of the reference and the dense wind scenarios on the evolution of a $1 M_{\odot}$ star; the rotational evolution model is described in Holzwarth \& Jardine (2005). The latter scenario ${ }^{4}$ is supposed to reflect both the approach and result of Wood et al. (2002, 2005a), that stellar mass losses scale with the wind density and increase strongly with the coronal X-ray flux/stellar rotation rate. For young stars, the CWRPs and mass loss rates based on the reference wind scenario are several orders of magnitude smaller than in the case of massive winds (Fig. 10). The cumulated mass loss is $\sim 10^{-4} M_{\odot}$, which supports the conjecture of Wood et al. (2002) that the young faint Sun-paradox cannot be explained through a higher-mass young Sun. The impact of stellar winds on planetary atmospheres and magnetospheres is most severe during the pre-main sequence phase (Fig. 11). The most promising targets for radio searches of extra-solar giant planets are thus rapidly rotating pre-main sequence stars, which do not only have strong winds but also high magnetic activity levels. We note that this conjecture is based on the extrapolation of the mainsequence wind scenarios to the pre-main sequence phase, since the wind ram pressures of young stars are as yet observationally unconstrained and thus hypothetical.

The magnetic energy flux at short-period orbits close to the star can be larger than the wind ram pressure (Figs. 11 and 1), which may entail different interaction mechanisms with planetary magnetospheres. Still closer to the star, Hot Jupiters are expected to interact directly with the coronal magnetic field. Magnetic reconnections between coronal field structures and the planetary magnetosphere alter the field topology and can initiate the onset of flares and chromospheric brightenings (Ip et al. 2004; Shkolnik et al. 2005). That close to the stellar corona, wind ram pressure effects are negligibly small (e.g. McIvor et al. 2006).

\subsection{Future issues}

It is essential to clarify the origin of the high CWRPs of the three $\mathrm{K}$ dwarfs $\epsilon$ Eri, $36 \mathrm{Oph}$, and $70 \mathrm{Oph}$, because they play the decisive role in the determination of a relationship between

${ }^{4}$ Since for the dense wind scenario with $n_{n}=5$, analysed in Sect. 3, the star loses about $65 \%$ of its initial mass within $20 \mathrm{Myr}$, we consider instead the milder case $n_{n}=2$ to illustrate the principal differences.

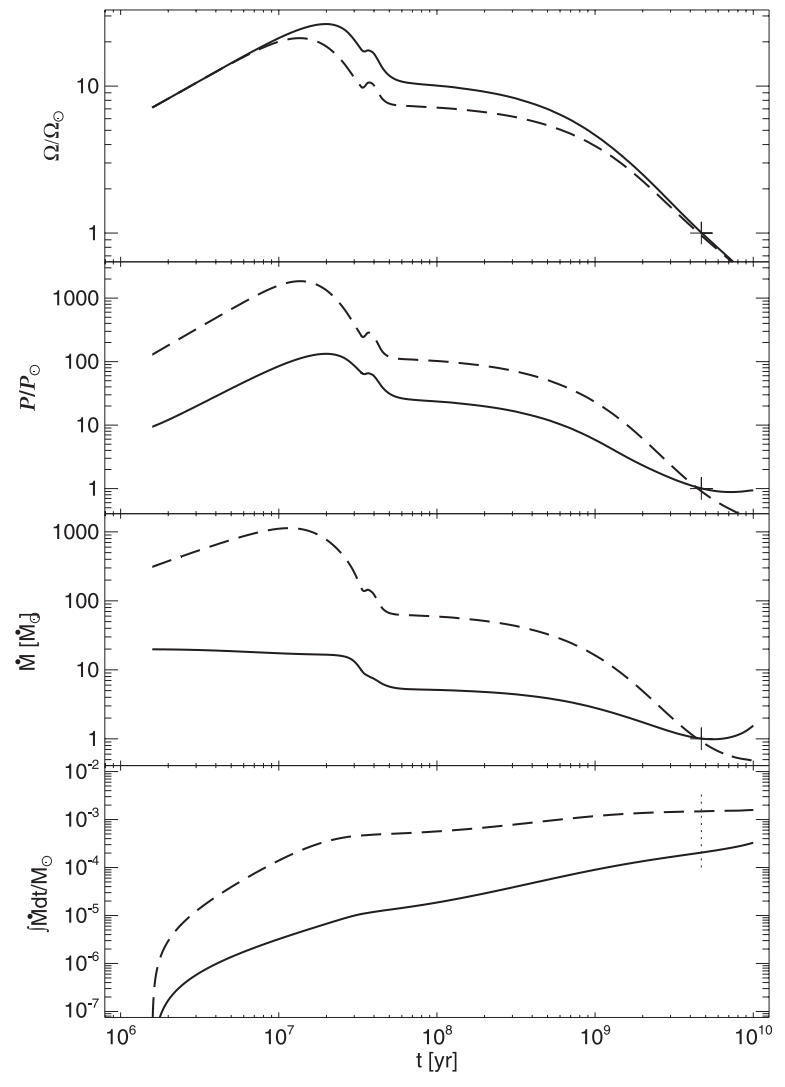

Fig. 10. Evolution of the stellar surface rotation rate, characteristic wind ram pressure, mass loss rate, and cumulative mass loss (top to bottom) of a $1 M_{\odot}$ star subject to wind scenarios with a moderate increase of thermal and magnetic wind parameters $\left(n_{T}=0.1, n_{n}=0.6, n_{\Phi}=1\right.$, solid lines) and with a strong increase of the wind density $\left(n_{T}=\right.$ $0.1, n_{n}=2, n_{\Phi}=1$, dashed lines), respectively. The initial rotation rate is $\Omega_{0}=2 \times 10^{-6} \mathrm{~s}^{-1}$, and the internal coupling timescale $\tau_{\mathrm{c}}=65 \mathrm{Myr}$. Crosses mark the current state of the Sun.

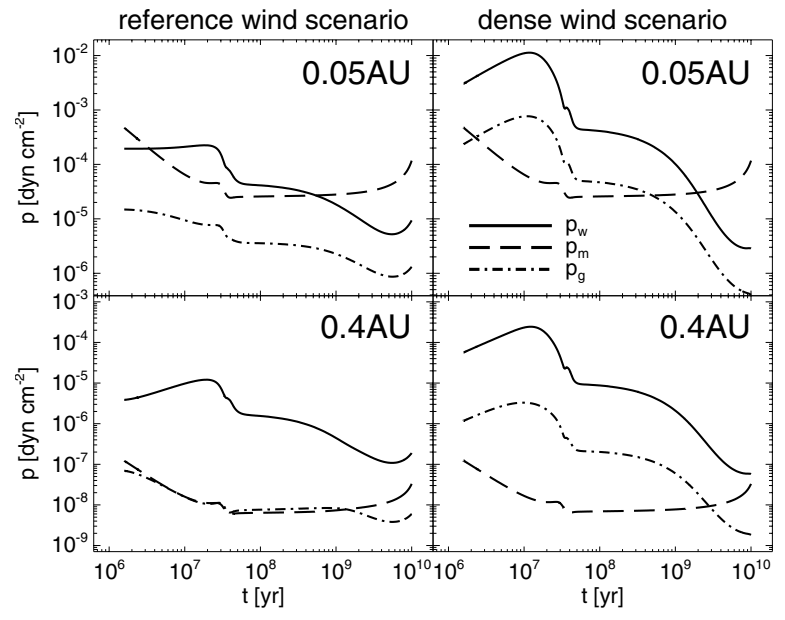

Fig. 11. Evolution of the wind ram pressure, $p_{\mathrm{w}}$, the magnetic pressure, $p_{\mathrm{m}}$, and the thermal gas pressure, $p_{\mathrm{g}}$, of magnetised winds at different distances inside the equatorial plane.

two fundamental stellar parameters. Since their wind ram pressures cannot be explained in terms of a solar-like wind model, we regard these stars as "non-representative" and excluded them from the comparison of empirical and theoretical values. This approach may formally account for the lower CWRP of the more rapidly rotating targets, but raises questions about 
inapplicabilities of the polytropic-magnetised wind model or/and power-law ansatz for the wind parameters. Wood et al., in contrast, disregard the empirical constraints set by the rapidly rotating targets and base their relationship on the high values of the K dwarfs. Their approach may formally account for the peculiar CWRPs, but raised the question about different wind properties and coronal field topologies beyond a certain stellar activity level. The present stellar sample is insufficient to conclusively decide between these two complementary approaches. More astrospherical detections of (preferentially single) main-sequence stars are highly desirable to solve this ambiguity.

If the high CWRP of the K dwarfs are observationally confirmed, then this will raise crucial questions about our current understanding of winds of cool stars, since the implicit requirements on the coronal properties are drastically different from what we know and expect from the Sun and solar-like stars. Although previous observations indicate otherwise, the most likely mechanism to account for such high wind ram pressure is an efficient magneto-centrifugal driving of the wind due to strong magnetic fields. Yet such high field strengths would raise the question how main sequence stars with rotation rates not much different from the Sun can produce and sustain such high magnetic flux densities over long times without modifying the coronal X-ray signatures.

\section{Conclusions}

Characteristic wind ram pressures and mass loss rates increase with the wind temperature, wind density, and strength of open magnetic fields. Albeit the observational data are as yet insufficient to conclusively discriminate between different wind scenarios, we argue in favour of a moderate increase of the thermal and magnetic wind properties with the stellar rotation rate and, synonymously, the coronal X-ray flux. Such a wind scenario does not account for the moderately rotating K dwarfs $\epsilon$ Eri, $36 \mathrm{Oph}$, and $70 \mathrm{Oph}$, whose observed thermal and magnetic properties do not allow for a consistent explanation of their high wind ram pressures in the framework of a magnetised wind model. We regard their high apparent mass loss rates as non-representative for cool main-sequence stars and suggest to exclude them from quantitative correlations between mass loss rates and rotation rates/coronal X-ray fluxes until their role is observationally and theoretically clarified.

The predicted rate of increase of mass loss rates is smaller than suggested by Wood et al. (2002, 2005a) and depends on whether a star can be classified as a slow or a fast magnetic rotator. In the latter case, efficient magneto-centrifugal driving of outflows entails terminal wind velocities considerably faster than the surface escape velocity, which, if not taken properly into account, leads to an overestimations of stellar mass loss rates. The predicted mass loss rates of cool main-sequence stars do not exceed (on average) about $10 M_{\odot}$. Since the predicted stellar winds are weaker than previously suggested, we expect less severe erosion of planetary atmospheres and lower detectabilities of magnetospheric radio emissions originating from extra-solar giant planets. Considering the evolution of stellar mass loss rates and wind ram pressure, we suggest that rapidly rotating pre-main sequence stars with high magnetic activity levels are the most promising targets for searches of planetary radio emission.

Acknowledgements. We thank the referee B. Wood for his very constructive comments which helped to improve the paper. V.H. gratefully acknowledges financial support for this research through a PPARC standard grand (PPA/G/S/2001/00144) and through a fellowship of the Max-Planck-Society.

\section{References}

Axford, W. I., \& Ip, W.-H. 1986, Adv. Space Res., 6, 27 Belcher, J. W., \& MacGregor, K. B. 1976, ApJ, 210, 498 Donahue, R. A., Saar, S. H., \& Baliunas, S. L. 1996, ApJ, 466, 384 Dupree, A. K., Brickhouse, N. S., Smith, G. H., \& Strader, J. 2005, ApJ, 625, L131

Favata, F., \& Micela, G. 2003, Space Sci. Rev., 108, 577

Gaidos, E. J., Güdel, M., \& Blake, G. A. 2000, Geophys. Res. Lett., 27, 501

Geiss, J. 1998, Space Sci. Rev., 85, 241

Grevesse, N., \& Sauval, A. J. 1998, Space Sci. Rev., 85, 161

Grießmeier, J.-M., Motschmann, U., Mann, G., \& Rucker, H. O. 2005, A\&A, 437, 717

Grießmeier, J.-M., Stadelmann, A., Penz, T., et al. 2004, A\&A, 425, 753

Güdel, M., Guinan, E. F., \& Skinner, S. L. 1997, ApJ, 483, 947

Holzwarth, V. 2005, A\&A, 440, 411

Holzwarth, V., \& Jardine, M. 2005, A\&A, 444, 661

Ip, W., Kopp, A., \& Hu, J. 2004, ApJ, 602, L53

Ivanova, N., \& Taam, R. E. 2003, ApJ, 599, 516

Jardine, M., Collier Cameron, A., \& Donati, J.-F. 2002a, MNRAS, 333, 339

Jardine, M., Wood, K., Collier Cameron, A., Donati, J.-F., \& Mackay, D. H. 2002b, MNRAS, 336, 1364

Kawaler, S. D. 1988, ApJ, 333, 236

Lamers, H. J. G. L. M., \& Cassinelli, J. P. 1999, Introduction to stellar winds (Cambridge University Press), ISBN 0521593980

Lim, J., \& White, S. M. 1996, ApJ, 462, L91

Marcy, G. W. 1984, ApJ, 276, 286

McIvor, T., Jardine, M., \& Holzwarth, V. 2006, MNRAS, 367, L1

Mestel, L. 1968, MNRAS, 138, 359

Mestel, L. 1999, Stellar magnetism (Oxford University Press)

Michel, F. C. 1969, ApJ, 158, 727

Müller, H.-R., Zank, G. P., \& Wood, B. E. 2001, ApJ, 551, 495

Nerney, S. 1980, ApJ, 242, 723

Noyes, R. W., Hartmann, L. W., Baliunas, S. L., Duncan, D. K., \& Vaughan, A. H. 1984, ApJ, 279, 763

Parker, E. N. 1960, ApJ, 132, 821

Pevtsov, A. A., Fisher, G. H., Acton, L. W., et al. 2003, ApJ, 598, 1387

Pizzolato, N., Maggio, A., Micela, G., Sciortino, S., \& Ventura, P. 2003, A\&A, 397,147

Priest, E. R., \& Pneuman, G. W. 1974, Sol. Phys., 34, 231

Raymond, J. C. 1999, Space Sci. Rev., 87, 55

Robinson, R. D., Worden, S. P., \& Harvey, J. W. 1980, ApJ, 236, L155

Rueedi, I., Solanki, S. K., Mathys, G., \& Saar, S. H. 1997, A\&A, 318, 429

Saar, S. H. 1991, in The Sun and cool stars: activity, magnetism, dynamos, IAU Coll., 130, ed. I. Tuominen, D. Moss, \& G. Rüdiger, Lect. Notes Phys. (Springer-Verlag), 380, 389

Saar, S. H. 2001, in 11th Cambridge Workshop on Cool Stars, Stellar Systems and the Sun, ed. R. J. Garcia Lopez, R. Rebolo, \& M. R. Zapaterio Osorio, ASP Conf. Ser., 223, 292

Saar, S. H., \& Osten, R. A. 1997, MNRAS, 284, 803

Sakurai, T. 1985, A\&A, 152, 121

Schrijver, C. J., DeRosa, M. L., \& Title, A. M. 2003, ApJ, 590, 493

Shaviv, N. J. 2003, J. Geophys. Res. (Space Physics), 108, 3

Shkolnik, E., Walker, G. A. H., Bohlender, D. A., Gu, P.-G., \& Kürster, M. 2005, ApJ, 622, 1075

Skumanich, A. 1972, ApJ, 171, 565

Stevens, I. R. 2005, MNRAS, 356, 1053

Stone, E. C., Cummings, A. C., McDonald, F. B., et al. 2005, Science, 309, 2017

Strassmeier, K. G. 2002, Astron. Nachr., 323, 309

Tsinganos, K., \& Bogovalov, S. 2000, A\&A, 356, 989

Valenti, J. A., Marcy, G. W., \& Basri, G. 1995, ApJ, 439, 939

van den Oord, G. H. J., \& Doyle, J. G. 1997, A\&A, 319, 578

Wang, Y.-M. 1998, in Cool Stars, Stellar Systems, and the Sun, ASP Conf. Ser., 154,131

Weber, E. J., \& Davis, L. J. 1967, ApJ, 148, 217

Withbroe, G. L., \& Noyes, R. W. 1977, ARA\&A, 15, 363

Wood, B. E. 2004, Living Rev. Sol. Phys., 1, 2

Wood, B. E., \& Linsky, J. L. 1998, ApJ, 492, 788

Wood, B. E., \& Linsky, J. L. 2006, ApJ, 643, 444

Wood, B. E., Müller, H., Zank, G. P., \& Linsky, J. L. 2002, ApJ, 574, 412

Wood, B. E., Müller, H.-R., Zank, G. P., Linsky, J. L., \& Redfield, S. 2005a, ApJ, 628, L143

Wood, B. E., Redfield, S., Linsky, J. L., Müller, H.-R., \& Zank, G. P. 2005b, ApJS, 159, 118

Zank, G. P., Pauls, H. L., Williams, L. L., \& Hall, D. T. 1996, J. Geophys. Res., 101,21639 